\title{
A COMPARISON OF LIGHTWEIGHT DESIGN CONCEPTS OF A PASSENGER AIRCRAFT SEAT USING TOPOLOGY AND CFRP LAMINATE OPTIMIZATION
}

\author{
Logan Kupchanko ${ }^{1}$, Stephen William Knox Roper ${ }^{1}$, Haksung Lee ${ }^{2}$, Mongyoung Huh ${ }^{2}$, Il Yong Kim ${ }^{1 *}$ \\ ${ }^{1}$ Department of Mechanical and Materials Engineering, Queen's University, Kingston, ON, Canada \\ ${ }^{2}$ Korea Institute of Carbon Convergence Technology, Jeonju, 54853, Korea \\ *Corresponding author: kimiy@queensu.ca
}

\begin{abstract}
The aerospace industry has significant motive to create lightweight structures to further the development of high efficiency aircraft. A topology optimization (TO) and laminate optimization approach to design a lightweight passenger seat concept was performed. Also, two types of discontinuous carbon-fibre reinforced plastics (CFRP), sheet molding compound (SMC) and long fiber prepreg sheet (LFPS) were compared in a case study against aluminum. discontinuous CFRP materials have undergone technological advancement and can pose as cost effective alternatives to continuous CFRP materials with acceptable performance loss. Optimization results were interpreted to form three lightweight design concepts which show notable weight saving potential. Among the three equal mass concepts, both discontinuous CFRP concepts showed 6-8\% lower compliance compared to aluminum, indicating potential for further weight reduction when using discontinuous CFRP material to meet the same stiffness requirements as a conventional aluminum design.
\end{abstract}

Keywords- topology optimization; laminate optimization; aircraft seat; lightweight; carbon sheet moulding compound

\section{INTRODUCTION}

Today's aerospace manufacturers have increased motivation to create higher efficiency aircraft for environmental and financial benefit. In creating higher efficiency aircraft, manufacturers strive to develop methods to create lightweight structures. This has been achieved through advanced design methods like topology optimization (TO) [1], and advanced materials such as carbon fibre reinforced plastics (CFRP) [2]. Utilizing advanced design methods and materials must be performed while sustaining safety and financial constraints. While high performance continuous CFRPs allow the highest structural advantage, industry has been reluctant due to their high material and manufacturing costs. A cost effective alternative to carbon fibre prepreg is discontinuous CFRP such as sheet molding compound (SMC) and long fiber prepreg sheet (LFPS). As carbon SMC and LFPS technology advances, similar performance can be achieved with lower cost compared to continuous CFRP.

Presented in this paper is an overview of a topology optimization approach to aircraft seat design as well as a laminate optimization technique utilizing Altair HyperWorks suite. In addition to optimization methods, two discontinuous CFRP materials will be investigated and compared to a conventional aluminum used for seat designs. A passenger seat was chosen as it has potential for high overall weight reduction of an aircraft due to the high repetition of parts.

\section{METHODOLOGY}

\section{A. Topology Optimization (TO)}

Topology optimization (TO) is a finite element based numerical design tool which determines an optimum distribution of material within a given design domain. TO has become an industry standard tool in developing high performance and lightweight structures in the aerospace industry, notably the design of aircraft wings and engine pylons [1]. TO uses a density based approach to determine the location of material that most significantly impact the user-defined objective and constraint functions, in this case compliance and mass-fraction respectively. Within the finite element model, compliance represents the global sum of element strain energies while mass fraction represents the user-defined ratio of material available for the solver to use during optimization, to the total in the initial design space. Practically, compliance can be thought of as a global measure of a structure's total internal deformation, or the inverse of stiffness. A structure having low compliance corresponds to having a high stiffness.

\section{B. Laminate Optimization}

Laminate optimization was performed using Altair HyperWorks Suite using a three stage optimization approach consisting of a free-size (thickness) optimization, discrete size optimization, and ply stacking sequence optimization. Initial ply bundles are created defining the initial ply materials, thickness distribution, and orientations. The ply bundles enter a free-size optimization which identifies the optimal ply shape and thickness of each ply bundle within the laminate. The second stage discretizes each ply bundle into individual plies and performs a size optimization determining the optimal thickness of each ply. Lastly a stacking sequence optimization determines the optimal order of individual plies within the finished laminate. Fig. 1 shows an overview of this process. 
(a)

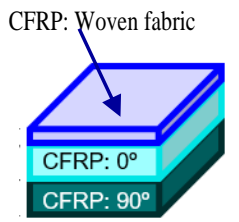

(b)

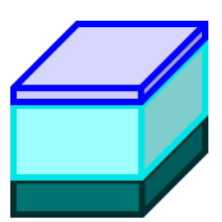

(c)

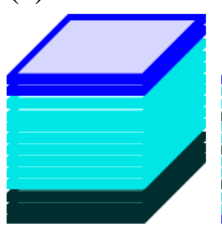

(d)

Figure 1: Laminate optimization process (a) initial ply bundle definition, (b) optimal ply bundle thickness post free-size optimization, (c) ply bundles separated into individual optimal thickness plies post discrete size optimization, and (d) stacking sequence post shuffling optimization

\section{MATERIALS AND MANUFACTURING PROCESSES}

\section{A. Sheet Moulding Compound and Long Fibre Prepreg Sheet}

Sheet moulding compound (SMC) is a composite material consisting of reinforcement material suspended within a matrix. Typically, the reinforcement material consists of fibres made from carbon or fiberglass and the matrix is a thermoset epoxy. Carbon SMC composites are manufactured by randomly dispersing chopped carbon fibres onto an epoxy covered film followed by a top layer of epoxy covered film [3]. Due to the random nature of fibres in SMC materials, they can often be classified as quasi-isotropic, having isotropic properties in the plane of the sheet. AMC-8590 is a chopped fibre sheet moulding compound consisting of $25 \mathrm{~mm}$ PAN based $12 \mathrm{~K}$ tow carbon fibre within a vinyl ester matrix [4]. LFPS is a material under development at KCTECH which is a long fibre prepreg sheet. Composed of 50mm long carbon fiber and epoxy resin system, LFPS was used to fabricate complex shape structures using hot compression molding [5]. AMC-8590 has a 53\% fibre weight fraction where LFPS has a $62 \%$ fibre weight fraction [4,6]. SMC and LFPS materials consist of near randomly oriented fibres; however, when used in compression moulding, the material flows into the shape of the final part, often allowing fibres to orient along part geometry.

\section{B. Compression Moulding Process}

The compression moulding process for carbon SMC and LFPS materials often consists of heated metal moulds which apply pressure and heat to the SMC and LFPS to form the shape and cure the epoxy simultaneously. Two variations of compression moulding will be examined; high flow moulding and low flow moulding which are shown in Fig. 2. High flow moulding typically utilizes a measured amount of bulk SMC material that gets compressed and drastically conforms to the shape of the mould. High flow allows a high amount of flow in the material which leads to high amount of flow-induced fibre alignment. Low flow moulding typically utilizes a stack of near-shape layers as the charge which requires less movement to conform to the shape of the mould. Low flow contributes to a low amount of flow-induced fibre alignment. Flow-induced fibre alignment often contributes to a higher tensile modulus and lower tensile strength [7]. Flow magnitude and direction heavily influence the anisotropy and mechanical properties of SMC materials, and accurate analysis requires a separate flow simulation to account for this uncertainty [8]. Material companies often provide mechanical properties for as-moulded and un-moulded specimens, to give insight to the magnitude of flow-induced fibre alignment. An example of this discrepancy is the provided mechanical properties of AMC-8590 where tensile modulus varies from $37 \mathrm{GPa}$ to $62 \mathrm{GPa}$ depending on manufacturing [4]. Unmoulded or 'machined' properties have lower strength and stiffness, and are recommended for engineering analysis.

For the purpose of TO where part shape is constantly changing, predicting flow characteristics would be infeasible and inaccurate. TO is a conceptual design tool, and for simplicity the lowest stated mechanical properties for each CFRP material were used. Mechanical properties are expected to improve after moulding, translating to a higher performing part than from optimization. Table I has mechanical properties for Aluminum 2024-T4, AMC-8590, and LFPS [4,6,9].

(a)

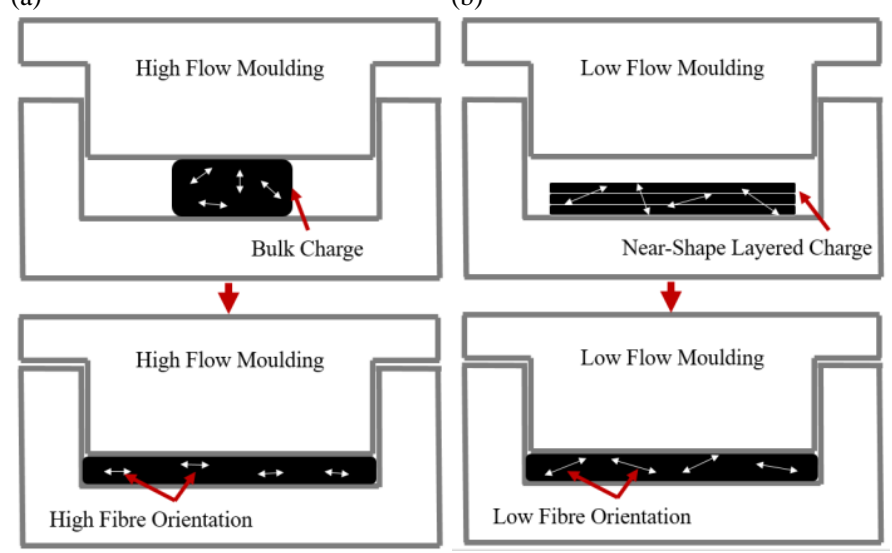

Figure 2: Compression moulding process showing (a) high flow moulding using a bulk material charge with high fibre alignment, and (b) low flow moulding using near-shape layered charge with low fibre alignment

TABLE I. MECHANICAL PROPERTIES OF MATERIALS USED IN TOPOLOGY AND LAMINATE OPTIMIZATION [4,6,9]

\begin{tabular}{|l|c|c|c|}
\hline \multirow{2}{*}{} & \multicolumn{2}{|c|}{$\begin{array}{c}\text { Topology } \\
\text { Optimization }\end{array}$} & $\begin{array}{c}\text { Laminate } \\
\text { Optimization }\end{array}$ \\
\cline { 2 - 4 } & $\begin{array}{c}\text { Aluminum } \\
\mathbf{2 0 2 4 - T 4}\end{array}$ & $\begin{array}{c}\text { AMC-8590} \\
\text { (moulded/machined) }\end{array}$ & $\begin{array}{c}\text { LFPS } \\
\text { (machined) }\end{array}$ \\
\hline Tensile Modulus [GPa] & 73 & $\begin{array}{c}62 / 37 \\
\text { ASTM D-638/D-3039 }\end{array}$ & $\begin{array}{c}38 \\
\text { ASTM D-3039 }\end{array}$ \\
\hline Tensile Strength [MPa] & 325 & $\begin{array}{c}276 / 162 \\
\text { ASTM D-638/D-3039 }\end{array}$ & $\begin{array}{c}346 \\
\text { ASTM D-3039 }\end{array}$ \\
\hline Density [g/cm $\left.{ }^{3}\right]$ & 2.78 & 1.48 & 1.46 \\
\hline
\end{tabular}

IV. MODELling

The quasi-isotropic nature of SMC and LFPS materials make ply orientation redundant in designing a laminate, simplifying the design and analysis procedure. For simplicity, the SMC material was assumed isotropic for TO. QuasiIsotropic mechanical properties were defined for the LFPS material in the laminate optimization.

Unconstrained TO tends to produce geometries which are difficult or impossible to manufacture, containing inaccessible tooling regions. Manufacturing constraints have been developed which restrict the allowable geometry produced by a TO result. For the aluminum seat design, a split draw direction manufacturing constraint was enforced to produce resulting geometry suited for casting or a two-setup machining operation. A split draw direction constraint enforces two draw directions normal to a selected plane, in this case down the 
center of each design domain. Manufacturing constraints typically reduce performance by enforcing less design freedom; however, the benefit of having a much closer to realistic designs [10]. Differences in performance are highly problem dependent and in some cases the effect of draw direction constraints is near negligible while producing much more interpretable results [10]. The AMC-8590 seat design features the use of a single draw direction constraint which produced results more compliant with the compression moulding process of SMC. There are several manufacturing constraints applied within the composite optimization. The resulting thickness of each ply bundle following the free-size optimization was discretized into individual plies according to a specified manufacturable ply thickness, for LFPS being 0.7 $\mathrm{mm}$. In all optimizations a minimum-dimension constraint was applied to enforce a minimum size of individual members to avoid infeasible, small features. The mathematical objective statements for the topology optimizations are as follows:

minimize

$$
C(\underset{\sim}{u}(\underset{\sim}{\rho}))={\underset{\sim}{f}}^{T} \underset{\sim}{u}(\underset{\sim}{\rho})
$$

subject to:

$$
\begin{aligned}
& \underset{\sim}{K u}=\underset{\sim}{f} \\
& \sum \rho_{e} V_{e} \omega_{e} / \sum V_{e} \omega_{e} \leq \overline{\mathrm{M}} \\
& \forall \text { element } e, \rho_{e} \in(0,1] \text { and } \\
& \left(0 \leq \rho_{i} \leq \rho_{j} \leq \ldots \leq \rho_{n} \leq 1\right)_{k}, k=1, \ldots, K
\end{aligned}
$$

where the compliance $C$ is the objective function; $\underset{\sim}{u}$ is the displacement vector; $\underset{\sim}{\rho}$ is the density vector; $f$ is the load vector; $\underset{\sim}{K}$ is the stiffness matrix; $\rho_{e}$ is the density of a given element $e ; V_{e}$ is the volume of a given element $e ; \omega_{e}$ is the material density of a given element $e ; \bar{M}$ is the user defined mass fraction; $\rho_{e} V_{e} \omega_{e}$ represents the mass of a given designable element during optimization; $V_{e} \omega_{e}$ represents the original mass of a given element.

Dimensions for the seat design domain were derived from SAE-ARP5526E and SAE-ARP5765A as well as work done by $\mathrm{M}$. Guida et al. [11-13]. The TO model consists of 1,049,920 designable hexahedral elements with average size of $2.5 \mathrm{~mm}$. The cross members are hollow steel tubes modeled using shell elements. Regions of load application, contact, and boundary conditions are non-designable and shown in grey in Fig. 3. The composite optimization model consists of 166,904 designable shell elements with an average size of $2.5 \mathrm{~mm}$. The cross members are modeled similarly to the TO model.

Linear static loading conditions were derived from inertial forces passengers would apply to the seat during ultimate

(a)

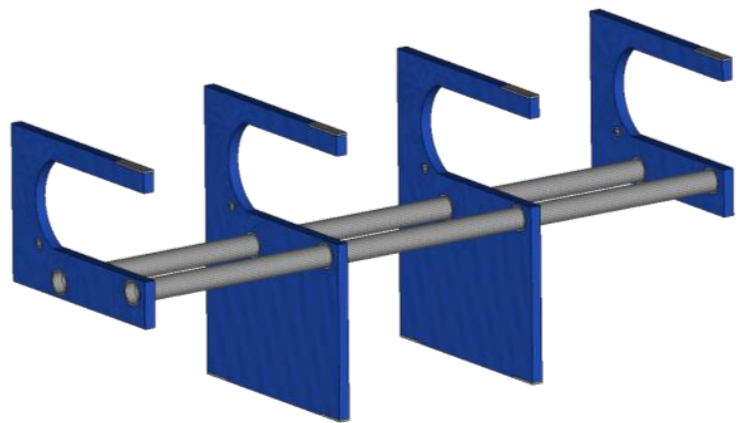

loading, crash scenarios, and general abuse loads from passengers. Ultimate load factors as defined in SAE-AS8049C are the maximum accelerations the aircraft is expected to endure during takeoff and landing, turbulence, or emergency maneuvers [14]. The structural requirements of an aircraft seat during ultimate loads is a no-yield condition. Crash scenarios are defined by SAE-AS8049C for passenger aircraft as a $16 \mathrm{G}$ forward crash with 10 degrees of yaw, and a $14 \mathrm{G}$ downward crash pitched 30 degrees forward [14]. Seat structures are allowed to yield during crash scenarios; however, no rupture, buckling, or large plastic deformation is allowed [14]. The abuse load considered in this analysis is a downward force of $1350 \mathrm{~N}$ on each armrest. Loading conditions are listed in Table II. Loads were applied to the model at locations guided by SAE-AS8049C using RBE3 interpolated elements to distribute loads onto the structure accordingly [14]. Crash loads are defined in terms of factors of gravity (G's) and are defined as an acceleration acting on a $77 \mathrm{~kg}$ occupant [14]. Eq. 1 is an example static load calculation from the $16 \mathrm{G}$ load factor to the load from a single passenger. Loads from three passengers are used in the analysis.

$$
\begin{gathered}
\operatorname{Mass}(\mathrm{kg}) \times \operatorname{load} \text { factor }(\mathrm{G}) \times \operatorname{gravity}\left(\mathrm{m} / \mathrm{s}^{2}\right)=\operatorname{static} \operatorname{load}(\mathrm{N}) \\
77(\mathrm{~kg}) \times 16(\mathrm{G}) \times 9.81\left(\mathrm{~m} / \mathrm{s}^{2}\right)=12,086(\mathrm{~N})
\end{gathered}
$$

TABLE II. LIST Of LOAD TYPE, MAGNITUDE, AND DiRECTION USED IN ANALYSIS [14]

\begin{tabular}{|c|c|c|c|}
\hline \multirow{2}{*}{ Load Type } & \multicolumn{2}{|c|}{ Load Magnitude } & Load Direction \\
\cline { 2 - 4 } & Load Factor [G] & Static Force [N] & - \\
\hline \multirow{3}{*}{$\begin{array}{c}\text { Ultimate } \\
\text { Loads }\end{array}$} & 3.0 & 2,266 & + Y dir. (Up) \\
\cline { 2 - 4 } & 1.5 & 1,133 & - Z dir. (Rear) \\
\cline { 2 - 4 } & 4.0 & 3,021 & + X dir. (Left) \\
\cline { 2 - 4 } Crash & 4.0 & 3,021 & - X dir. (Right) \\
\cline { 2 - 4 } Scenarios & 16.0 & 12,086 & + Z dir. (10 degrees yaw) \\
\cline { 2 - 4 } Abuse Load & 14.0 & 10,575 & - Y dir. (30 degrees pitch) \\
\hline
\end{tabular}

\section{RESUlTS}

TO and laminate optimization yielded results summarized in Table III with geometries shown in Fig. 4. A mass of $10 \mathrm{~kg}$ was set for the designable material after optimization. The aluminum results were taken as a baseline with both CFRP results having lower compliance values. The TO result using AMC-8590 had a 5.8\% lower compliance value compared to the aluminum TO results. Laminate optimization results for LFPS material show a higher performance benefit with a compliance value $8.0 \%$ lower than the aluminum TO solution. Both CFRP solutions had similar results within around $2 \%$ of each other despite different optimization techniques.

(b)

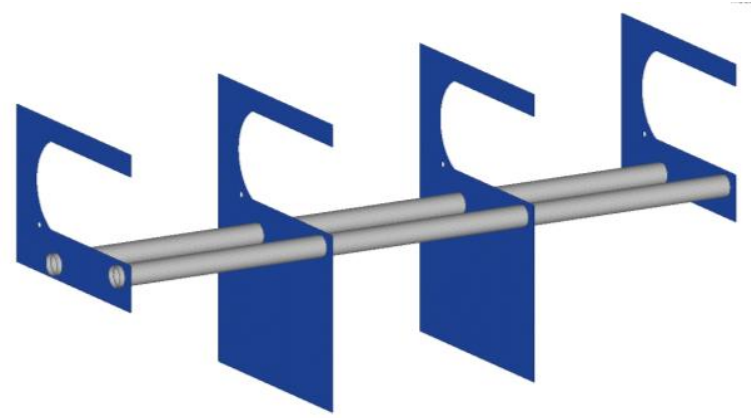

Figure 3: Finite element models for (a) topology optimization, and (b) laminate optimization. Design space shown in blue, non-design space shown in grey. 
(a)

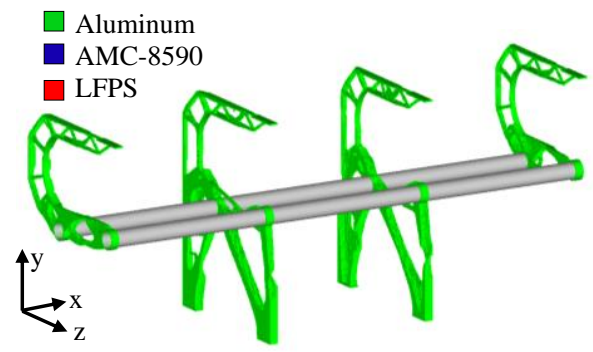

(b)

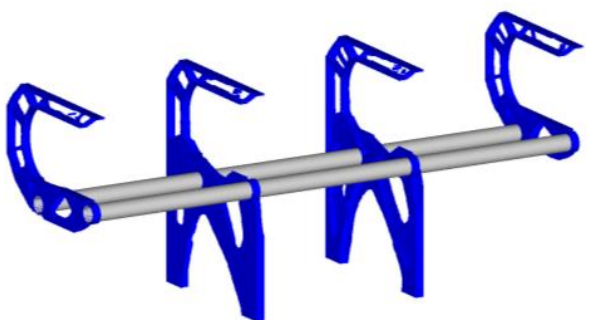

(c)

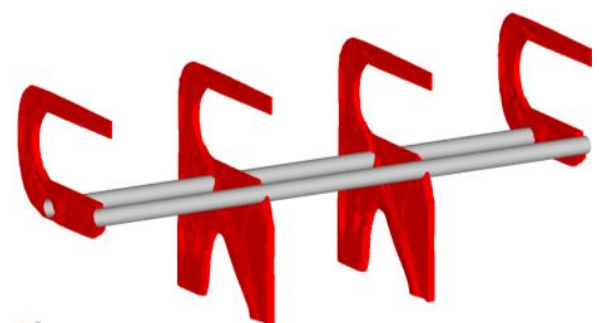

Figure 4: Initial optimization results for aircraft passenger seat design. (a) TO with aluminum. (b) TO with AMC8590. (c) laminate optimization with LFPS.

TABLE III. OPTIMIZATION RESUlTS COMPARING PERFORMANCE METRICS OF COMPLIANCE AND MAXIMUM STRESS

\begin{tabular}{|c|c|c|c|}
\hline \multirow{2}{*}{ Load Type } & \multicolumn{2}{|c|}{ Topology Optimization } & $\begin{array}{c}\text { Laminate } \\
\text { Optimization }\end{array}$ \\
\cline { 2 - 4 } & Aluminum 2024 & AMC-8590 & LFPS \\
\hline $\begin{array}{c}\text { Design Space } \\
\text { Mass [kg] }\end{array}$ & $\begin{array}{c}10.0 \\
\text { (baseline) }\end{array}$ & $\begin{array}{c}10.0 \\
(\mathrm{~N} / \mathrm{A})\end{array}$ & $\begin{array}{c}10.0 \\
(\mathrm{~N} / \mathrm{A})\end{array}$ \\
\hline $\begin{array}{c}\text { Design Space } \\
\text { Compliance [J] }\end{array}$ & $\begin{array}{c}109.8 \\
\text { (baseline) }\end{array}$ & $\begin{array}{c}103.4 \\
(-5.8 \%)\end{array}$ & $\begin{array}{c}101.0 \\
(-8.0 \%)\end{array}$ \\
\hline $\begin{array}{c}\text { Maximum } \\
\text { Stress [MPa] }\end{array}$ & 305 & 206 & 379 \\
\hline $\begin{array}{c}\text { Yield Safety } \\
\text { Factor }\end{array}$ & 1.06 & 0.78 & 0.91 \\
\hline
\end{tabular}

All areas of maximum stress were located surrounding the seatbelt attachment points, where the majority of the loading is applied. It is common in practice to add metal inserts into SMC components to add strength to fastener locations [15]. In the AMC-8590 topology optimization the non-designable regions were modeled as aluminum to represent aluminum inserts to reinforce the load application points. In all three models the max stress occurred during the $16 \mathrm{G}$ crash loading with small amounts of local yielding occurring near seatbelt attachment points as shown in Fig. 6. The structures only showed signs of yielding during the 16 $\mathrm{G}$ crash scenario, where by specification, yielding is allowed [14]. Both CFRP concepts had safety factors to material yielding below 1, indicating that yielding had occurred. These safety factors were based upon the minimum mechanical strength values published by manufactures and is likely to improve if more accurate mechanical properties are determined through mould flow modelling.

Fig. 5 shows a detailed example of the final laminate results obtained through the laminate optimization. The resulting geometry is discretized into $0.7 \mathrm{~mm}$ thick plies.

The TO objective was to minimize compliance subject to a mass constraint, meaning both results would have equal mass for comparison. When comparing the compliance value of the AMC-8590 result to aluminum, a 5.8\% stiffer structure was produced. To put these results in a more meaningful perspective, this means that a lighter CFRP structure could be designed to the same level of stiffness as a conventional aluminum design.
Both SMC and LFPS concepts show similar performance in relation to the stiffness of the structure. This is partly due to similar elastic moduli in the un-moulded or machined state, $37 \mathrm{GPa}$ and $38 \mathrm{GPa}$ for AMC-8590 and LFPS, respectively. Both $\mathrm{TO}$ and laminate optimization found local optimum geometries with comparable performance values. LFPS has a significantly higher tensile strength compared to AMC-8590 in the un-moulded state, $346 \mathrm{MPa}$ and $162 \mathrm{MPa}$ respectively. This could make the use of LFPS more desirable when subject to meeting the design criteria during crash simulation. For a more accurate comparison of performance, mould design and flow modeling to predict flow-induced fibre alignment would need to be performed. This analysis has shown that using carbon fibre SMC or LFPS material would allow for a lighter weight seat structure when compared to using aluminum.

Future work will include explicit dynamic simulation using the procedure outlined in SAE-AS8049C [14]. LSDyna software will be used with anthropomorphic test dummy (ATD) models for higher accuracy results compared to the linear-static loading performed for concept design in this work.

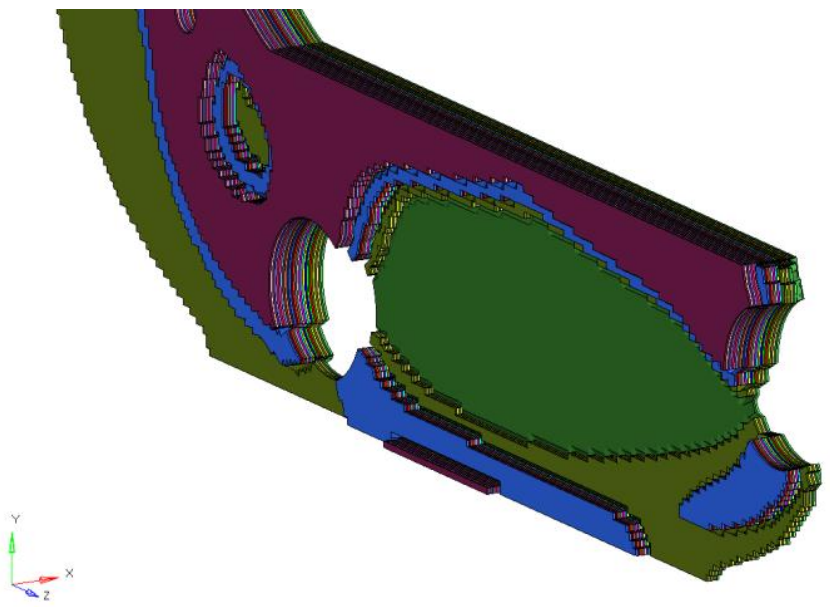

Figure 5: Detailed view of optimized laminate of LFPS for the lower portion of the armrest structure. 
(a)

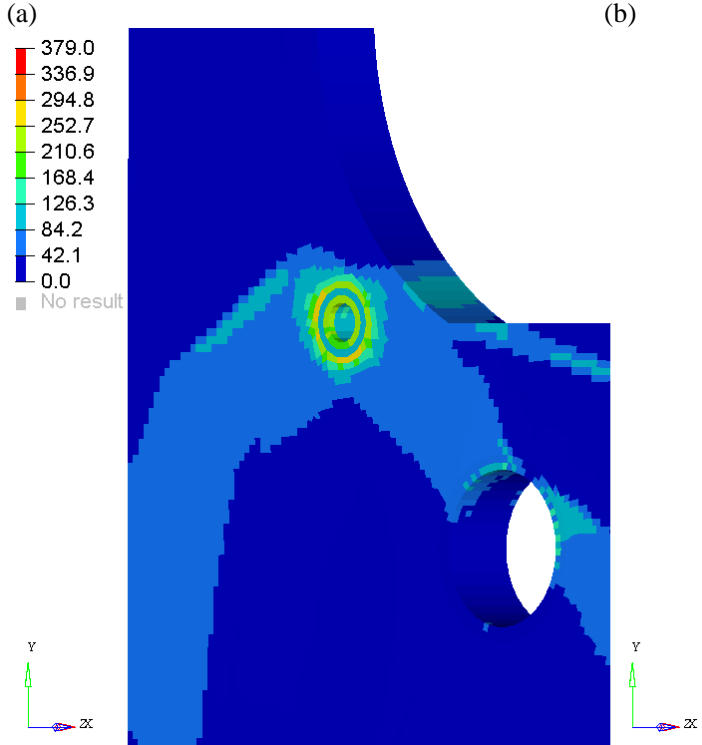

Figure 6: Stress contour plot for the $16 \mathrm{G}$ crash scenario showing

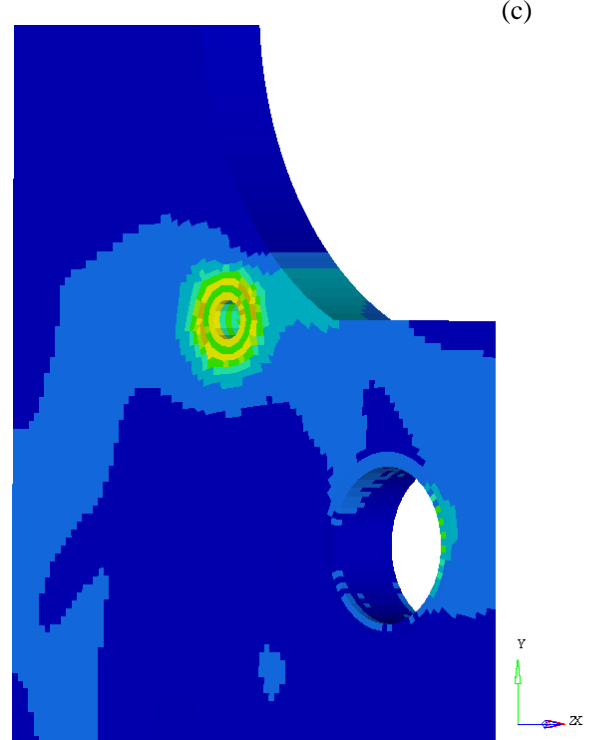

(c)

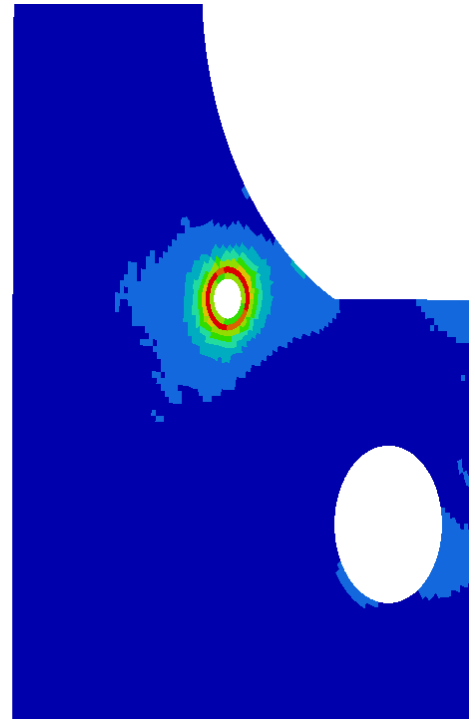

(c) LFPS composite model
(c)

\section{ACKNOWLEDGMENT}

This research was funded by the Natural Sciences and Engineering Research Council of Canada (NSERC) and The Korea Institute of Carbon Convergence Technology (KCTECH).

\section{REFERENCES}

[1] J.H. Zhu, W.H. Zhang, and L. Xia, "Topology Optimization in Aircraft and Aerospace Structures Design," Arch Computat Methods Eng (2016) 23: 595-622.

[2] F. W. J. van Hattum, F. Regel, and M. Labordus, (2011) Cost reduction in manufacturing of aerospace composites, Plastics, Rubber and Composites, 40:2, 93-99, DOI $10.1179 / 174328911$ X12988622801052

[3] H. Lee, M. Huh, J. Yoon, D. Lee, S. Kim, and S. Kang, "Fabrication of carbon fiber SMC composites with vinyl ester resin and effect of carbon fiber content on mechanical properties," Carbon Letters Vol. 22, 101-104 (2017)

[4] A. Schulman, "Quantum Composites Material Summary" (2017).

[5] J.-H. Bae, M.-G. Han, and S.-H. Chang, "Formability of complex composite structures with ribs made of long carbon-fiber-reinforced prepregs," Composite Structures, vol. 168, pp. 56-64, 2017.

[6] Korea Institute of Carbon Convergence Technology (KCTECH)

[7] C. Nony-Davadie, L. Peltier, Y. Chemisky, B. Surowiec, and F. Meraghni, "Mechanical characterization of anisotropy on a carbon fiber sheet molding compound composite under quasi-static and fatigue loading," Journal of Composite Materials - Vol. Octobre, p.21p. -2018

[8] Y. Li, Z. Chen, H. Xu, J. Dahl, D. Zeng, M. Mirdamadi, and X. Su, "Modeling and simulation of compression molding process for sheet molding compound (SMC) of chopped carbon fiber composites," SAE Int. J. Mater. Manf. 10(2):2017, doi:10.4271/2017-01-0228.

[9] Metals Handbook, Vol.2 - Properties and Selection: Nonferrous Alloys and Special-Purpose Materials, ASM International $10^{\text {th }}$ Ed. 1990.

[10] M. Zhou, R. Fleury, Y.K. Shyy, H. Thomas, and J.M. Brennan, "Progress in Topology Optimization with Manufacturing Constraints," AIAA/ISSMO (2002).
[11] Aerospace Reccomended Practice: ARP5526 Rev. E “Aircraft Seat Design Guidelines and Clarifications", SAE International (2018).

[12] Aerospace Reccomended Practice: ARP5765 Rev. A "Analytical Methods for Aricraft Seat Design and Evaluation", SAE International (2015).

[13] M. Guida, A. Manzoni, A. Zuppardi, F. Caputo, F. Marnulo, and A. De Luca, "Development of a multibody system for crashworthiness certification of aircraft seat," Multibody Syst Dyn (2018) 44:191-221

[14] Aerospace Standard: AS8049 Rev. C "Performance Standard for Seats in Civil Rotorcraft, Transport Aircraft, and General Aviation Aircraft", SAE International (2015)

[15] M. Fette, M. Hentschel, F. Kohler, J. Wulfsberg, and A.Herrmann, "Automated and cost-efficient Production of Hybrid Sheet Moulding Compound Aircraft Components," Procedia Manufacturing 6 (2016) 132-139. 